\title{
HTwitt: a hadoop-based platform for analysis and visualization of streaming Twitter data
}

\author{
Umit Demirbaga $^{1,2}$ (D) \\ Received: 15 October 2020/Accepted: 15 April 2021 \\ (C) The Author(s) 2021
}

\begin{abstract}
Twitter produces a massive amount of data due to its popularity that is one of the reasons underlying big data problems. One of those problems is the classification of tweets due to use of sophisticated and complex language, which makes the current tools insufficient. We present our framework HTwitt, built on top of the Hadoop ecosystem, which consists of a MapReduce algorithm and a set of machine learning techniques embedded within a big data analytics platform to efficiently address the following problems: (1) traditional data processing techniques are inadequate to handle big data; (2) data preprocessing needs substantial manual effort; (3) domain knowledge is required before the classification; (4) semantic explanation is ignored. In this work, these challenges are overcome by using different algorithms combined with a Naïve Bayes classifier to ensure reliability and highly precise recommendations in virtualization and cloud environments. These features make HTwitt different from others in terms of having an effective and practical design for text classification in big data analytics. The main contribution of the paper is to propose a framework for building landslide early warning systems by pinpointing useful tweets and visualizing them along with the processed information. We demonstrate the results of the experiments which quantify the levels of overfitting in the training stage of the model using different sizes of real-world datasets in machine learning phases. Our results demonstrate that the proposed system provides high-quality results with a score of nearly $95 \%$ and meets the requirement of a Hadoop-based classification system.
\end{abstract}

Keywords Big data $\cdot$ MapReduce $\cdot$ Machine learning $\cdot$ Classification $\cdot$ Monitoring $\cdot$ Visualization

\section{Introduction}

The concept of big data has become a part of our lives with the use of the internet although it first appeared in astronomy and genetics $[1,2]$. The fact that the computer is in almost every area of our lives has resulted in the storage, processing, and management of a huge amount of data. The use of the internet in many areas of our lives has also increased the amount of data available in electronic form by the help of cloud computing [3,4]. The data we are talking about, which consists of the data entered and stored

A preliminary version of this paper is presented in IEEE SC2 2018 Conference.

Umit Demirbaga

u.demirbaga2@ncl.ac.uk

1 School of Computing, Newcastle University, Newcastle upon Tyne, UK

2 Faculty of Engineering, Bartin University, Bartin, Turkey to perform services along with a huge amount of data that is mostly useless, is growing like an avalanche. With 2019 figures, more than 2.5 quintillion bytes of data are generated in a day in the world, and the data size is estimated to reach 45 times that in 10 years [5]. So, we see a garbage dump consisting of unstructured data. It was not long before it was understood that this phenomenon, called 'information dump', was potentially a large treasure but could not be used because it was not structured. As a matter of fact, this dump, which consists of items such as posts on social media platforms, blogs, records of web servers, internet statistics, can be converted into a useful and beneficial form. If these data are interpreted with the correct analytics methods, a great progress will be made in making important decisions, managing risks correctly and signposting new discoveries. For these crucial reasons, big data analytics is getting more and more important in some special areas, such as government sector [6], e-commerce [7, 8], semantic analysis [9], healthcare industry [10-12], entertainment industry [13], weather patterns [14], linear 
road benchmarking [15], banking sector [16, 17], IoT technology [18-20], natural disaster management [21], cyber-physical systems [22, 23], smart city projects [24-26], and social networks [27, 28].

In recent years, social media has generated tons of data every second as it becomes universally accepted. Twitter, one of the most widespread social networks, has led to the generation of huge data, as it allows people to share their thoughts easily everywhere about any topic. At the same time, it is a major area of interest within the field of gaining precious information. Twitter provides Rest API for developers to retrieve tweets and related information and Streaming API for those who are curious about real-time data [29]. In this way, very big data sets can be collected, analyzed, and stored in different data formats. However, advanced processing techniques are required for such operations due to the big data characteristics known as the $5 \mathrm{~V}$ model: volume, variety, velocity, variability, veracity [30, 31]. In addition, processing and analysis of Twitter data are remarkably complex and difficult in comparison to traditional and well-formatted sets of documents as the tweets do not always contain an actual "pass value". For these reasons, it is not possible to process big, complex and streaming data with traditional methods. Hadoop has the capability to handle all kinds of data in petabyte size that requires cloud-based data centers [32], and comes with some considerable features, such as scalable, cost-effective, fast, resilience to system failures. The MapReduce technique, one of Hadoop's main components, provides an appropriate method to analyze Twitter big data [33]. Apache Mahout provides different machine learning algorithms, and runs on top of Apache Hadoop [34]. That is the reason why the MapReduce algorithm is convenient to apply machine learning techniques on the Twitter social media platform. The combination of MapReduce paradigm and the machine learning library provides the opportunity of extracting beneficial information from large-scale databases.

In this paper, we introduce HTwitt, a Hadoop-based platform for analysis and visualization of streaming Twitter data, which analyzes and visualizes tweets using big data technologies along with a set of machine learning algorithms to address the following problems: (1) traditional data processing techniques cannot work for big data; (2) manual intervention and efforts are inadequate for preprocessing of big data; (3) tweets are generally noisy and contain a lot of nonsense phrases that make it difficult to extract useful expressions. HTwitt analytics lifecycle proposes the process of conducting data analysis for both batch and streaming for text mining applications. This work, therefore, is one step ahead of other works in terms of effectiveness and usefulness by means of its flexible and modular design. In text classification, machine learning is used to make classifications by learning from past observations rather than resorting to manually designed rules. A machine learning algorithm learns the different relationships between the pieces of text using pre-labelled examples for training purposes $[35,36]$. It gives expected outputs (i.e., tags) to pieces of unlearned text using these learned patterns (i.e., text). Last but not least, machine learning teaches machines to understand and act without being directly programmed that significantly affects the performance of rule-based applications as it learns the patterns of the complexity of the problem before. The purpose of using machine learning in this study is to develop a scalable and efficient algorithm with tweets accumulated over time and automatically classify streaming tweets. In HTwitt framework, the data are first parsed and transferred into csv format, then classified using the MapReduce algorithm based on the location where the tweets were posted from and post date and time in the Data Preparation module. These outputs are used for training and testing a model for classifying tweets related to natural disaster in the Model Training and Testing system using the Naïve Bayes machine learning algorithm to perform an efficient classification as the British Geological Survey (BGS) collects tweets related to landslide event which includes the words of landslide, soil sliding, mudslide, landfall, landslip. We use a Naïve Bayes classifier as it is easy to implement and does not require as much data for training, as well as not being prone to irrelevant features. We propose this framework to distinguish the tweets related to natural events from the tweets which involve landslide phrase but are not related to the natural events in some sentences, as many irrelevant tweets were also gathered during the data collection from Twitter due to homophones. For example, in politics, the landslide expression means an overwhelming victory in the elections as well. In addition to that, we created the Real-time data collection/injection module to acquire the streaming data from Twitter based on the specific keywords using Twitter Streaming API and to store them into Hadoop Distributed File System (HDFS) concurrently. However, Twitter has a restriction on streaming API, which allows users to download a limited number of tweets at a specified time. For this reason, an efficient tool is required to retrieve very big data from Twitter. That is why we decided to use Apache Flume, a highly distributed, reliable, and configurable tool, to collect, aggregate and move huge amounts of data. To visualize the result of the predicted decisions after classifying the tweets, visualization module allows users to see the exact location of the tweets. These features make our framework different from others in terms of processing and analyzing large Twitter data. 


\subsection{Related work}

Over the last few years, there has been a rising consideration in the area of Twitter sentiment analysis within Hadoop ecosystems. Beck et al. [37] proposed a recommender system using item-based Collaborative Filtering algorithms and implementing it using Apache Mahout. The system processes the streaming messages by splitting them into small pieces and then builds a recommendation model for news recommendation. Meanwhile, the system continues to build a new recommender and replaces the old model with the new one. The results of the experiments show that the proposed system ensures highly precise results and low response time. Tare et al. [38] presented a system to classify a large number of tweets using Naïve Bayes algorithm over a MapReduce paradigm. They categorize the tweets based on their topics using a multi-node Hadoop cluster. The main purpose of the work is to show how to combine Naïve Bayes algorithm with MapReduce algorithm. Martin et al. [39] proposed a framework implemented in Apache Spark to describe city events and explore their spatial-temporal characteristics by analyzing the tweets. They conducted this study in the city of Valencia, Spain and focus on how Twitter data can be used to depict city events and explore its spatial-temporal properties. A general event map was created by using positive and negative emotions in determining the location of an event by using a one-year daily average heat map of the city. At the end of the study, they combine all daily events in a single map to create a convenient urban infrastructure of Valencia city. The authors in [40] propose a recommendation system implemented in Apache Mahout to provide users' reviews, feedback, opinions and comments regarding any items using a large amount of web data. They use a set of filtering techniques along with classification algorithms to extract positive and negative reviews about movies. Yang et al. [41] used a supervised classification approach to identify adverse drug reactions automatically through a dimension reduction technique called Latent Dirichlet Allocation (LDA). They collect 3000 original posts and comments about people's drug use experience over 60 days via a Web spidering program and use this data to build a robust classifier. They claim that the performance of the proposed method is quite satisfactory in identifying drug surveillance. The authors in [42] proposed a model to analyze unstructured social media datasets obtained from Twitter for sentiment analysis using both supervised and unsupervised machine learning methods. They achieved $80.68 \%$ accuracy on the unsupervised method implementing Support Vector Machines, Multinomial Naïve Bayes, and Maximum Entropy while achieving an accuracy of $67 \%$ on the supervised method by performing unigram, bigram and Part-of-Speech techniques. Authors in [43] proposed a Hadoop-based framework implemented in MapReduce programming model to extract the customers' opinion about the products to inform the companies. They collected tweets via Twitter $4 \mathrm{~J}$ and stored them in HDFS through Apache Flume. Then, they performed the sentiment analysis via SentiGem sentiment analysis API by classifying the words as negative, positive and neutral opinion. Khade [44] used a C4.5 decision tree algorithm by combining it with a MapReduce paradigm to create a model which predicts customer behavior as they claim that traditional decision tree algorithms are not convenient for big data. They used a data set which contains a large number of customer records related to purchases. They successfully analyzed the new data using the created model and stored the results in HDFS. They finally visualized the data using D3.js through a web user interface.

However, these works are not sufficient for providing an adaptive and time-efficient framework for both streaming and batch big Twitter data analysis. Table 1 gives a summary of other related papers along with their specifications and limitations.

\subsection{Motivation}

Although some researchers have developed frameworks to refer to this area, there is still a gap in the literature concerning a comprehensive social media analysis [55]. The authors in [56] define four basic steps of social media analytics in comprehensive coverage: Discovery, revealing of hidden structures; Tracking, which includes decisions about the data source (e.g., LinkedIn, Instagram, Twitter), approach and method; Preparation, which explains the steps to make the data ready for processing; and finally Analysis, which covers a variety of methods, including opinion mining. In [57], the key factors of the difference between this new fact and the traditional analytics are summarized as $3 \mathrm{~V}$ : Velocity, Volume and Variety, which causes some problems on working with Twitter data, such as (1) Retrieving data sets in real-time; (2) Extracting useful information; (3) Getting meaningful outputs; (4) Distinguishing the tweets based on the contents of the text; (5) Visualizing the tweets along with the selected parameters. Different frameworks are proposed in the literature. However, to the best of our knowledge, there is no previous work addressing all the challenges above while proposing a comprehensive framework for an automated classification of Twitter messages into categories with a high accuracy without intervention from a human operator and visualization of the outputs in a very short period of time. This work contributes to designing a highly available, scalable and cost-effective framework for text classification in big 
Table 1 Summary of other related papers

\begin{tabular}{llllll}
\hline Study & Analysis & Methods & Processing types & Data usage & Visualization \\
\hline$[45]$ & MapReduce & Naïve Bayes algo & Batch & Classification & No \\
{$[46]$} & MapReduce & n-Gram language model & Batch & Decision support & \\
& & K-nearest neighbors algo & & No \\
{$[47]$} & MapReduce & n-Gram language model & Batch & Event detection \\
{$[48]$} & MapReduce & K-means algo & Batch & Clustering & No \\
& & Particle swarm opt. & Real-time & Early detection \\
{$[49]$} & Spark & Random forest algo & Real-time & Disease detection \\
{$[50]$} & Spark & Naïve Bayes algo & Yogistic regression & Batch & Spam detection \\
{$[51]$} & MapReduce & Approx. string matching & Batch & Personality extraction \\
{$[52]$} & MapReduce & Five-factor model & Batch & Clustering & No \\
{$[53]$} & Hive & K-means algo & Batch & Clustering & No \\
\hline 54$]$ & MapReduce & Clustering algo & &
\end{tabular}

data analytics. This research, therefore, shows the usefulness of the proposed framework that has many significant advantages in terms of agility and flexibility along with the accuracy of the analysis results that makes it one step ahead of the other works, especially for early warning systems.

\subsection{Contributions}

In this paper, we aim to build a novel framework, HTwitt, which allows users to collect, analyze and visualize a large amount of Twitter data using a set of machine learning algorithms in big data ecosystem. The contribution of this paper is to develop the framework of the previous work [58] to carry out the following scenarios:

- Proposing a novel framework for batch and stream processing pipelines for smart early warning systems.

- The collection of real-time data from Twitter and storing them into HDFS automatically without manual intervention.

- Combining a set of techniques and algorithms with the big data frameworks to analyze large-scale Twitter data.

- Defining the key factors that impact the performance of the analysis of Twitter data, such as the levels of overfitting in the training stage of the model using realworld data sets in different size and the varying execution time of the model training with different CPU numbers based on the ideal data size.

- The visualization of the analyzed results on Google Earth.

Outline. A brief background is given in Sect. 2. In Sect. 3, the implementation of our proposed framework is presented. Section 4 illustrates the experimental results and discussion while Sect. 5 concludes this paper discussing the conclusion and future work.

\section{Background}

The rapid growth of social media has caused some serious issues regarding data storage and management, which has led to the emergence of big data framework technologies as the classic data process methods are insufficient to handle such data. The big data frameworks used in this work are Apache Hadoop, ${ }^{1}$ Apache Mahout, ${ }^{2}$ Apache Flume ${ }^{3}$ along with a set of machine learning techniques presented in the next section. Apache Hadoop enables the processing of a huge amount of data, both batch and streaming, and implements MapReduce paradigm as a run-time system [59] while Apache Mahout allows developers to implement their machine learning algorithm by providing a set of classification algorithms on a distributed cluster [60]. Apache Flume has the ability to collect, aggregate, and move large amounts of streaming data from various sources to a centralized data storage like HDFS [61].

\subsection{Preliminaries}

In this section, important background concepts are given briefly, including Naïve Bayes classifier, n-Grams language model, Tf-Idf, Log-Likelihood Ratio Similarity, and Normalization to develop the proposed model.

\footnotetext{
${ }^{1}$ https://hadoop.apache.org/.

${ }^{2}$ https://mahout.apache.org/.

3 https://flume.apache.org/.
} 


\subsubsection{Naïve Bayes classifier}

Naïve Bayes is a well-known probabilistic classification algorithm that proposes solutions for classification problems [62]. It is used for classification purpose through a set of given predictors trained with training data that creates a classifier by calculating the posterior probability based on the attributes.

Equation 1 is used to calculate probability based on class conditional independence in a Naïve Bayes algorithm.

$P(x / y)=\frac{P(y / x) * P(x)}{P(y)}$

where $P(x / y)$ represents the probability of event $\mathrm{x}$ occurring when event $\mathrm{y}$ is given. $P(x)$ shows the probability of occurrence of $\mathrm{x}$ while $P(y)$ indicates the probability of occurrence of $y$. Lastly, $P(y / x)$ states the probability of event $y$ occurring when event $x$ is given.

\subsection{2 n-grams language model}

n-Grams models, termed as a series of words, are commonly used in statistical natural language processing [63]. Words are modelled so that each n-Gram consists of $n$ words for parsing. Each separate word is named as 1-Gram. Two words, like happy day, are regarded as a single unit and called 2-Grams (bigram). Figure 1 shows a sample of $\mathrm{n}$-Grams for three different models.

Equation 2 demonstrates the calculation of the n-Grams language model.

$p\left(w_{i} \mid w_{1}, \ldots, w_{i-1}\right)=p\left(w_{i} \mid w_{i-n+1}, \ldots, w_{i-1}\right)$

Here, $p\left(w_{i}\right)$ states unigram and $p\left(w_{i} \mid w_{i-1}\right)$ symbolizes $b i$ gram while $p\left(w_{i} \mid w_{i-2}, w_{i-1}\right)$ indicates trigram [64].

When $f$ is considered as a word, then

$w_{f}=\frac{N_{f}}{\operatorname{count}(f)}$

where $f$ indicates the weight in the feature vector while $N_{\mathrm{f}}$ states how many times $f$ appeared in the tweet and finally $\operatorname{count}(f)$ shows the quantity of $f$ value.

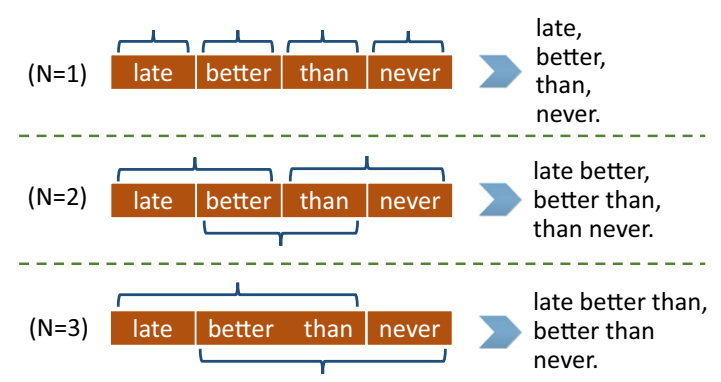

Fig. 1 A sample of n-Grams models

\subsubsection{Tf-Idf}

Tf-Idf (term frequency-inverse document frequency) is a text processing algorithm usually used in information retrieval (IR) that reflects how relevant a keyword is in a given document and assigns the importance to that keyword depending on how many times it appears in the document [65]. While the $T f$ represents how many times a word is in a text, the Idf calculates how significant that phrase is in the entire corpus. The calculations of $t f i$, idfi and $t$ fidf are shown in Eqs. 4, 5 and 6, respectively.

$$
\begin{aligned}
& \operatorname{tf}_{i}=\frac{\left(n_{i}\right)}{\sum_{k} n_{k}} \\
& \operatorname{idf}_{i}=\log \frac{|D|}{\left|d: t_{i} \in d\right|} \\
& \text { tfidf }=\mathrm{tf} * \text { idf }
\end{aligned}
$$

\subsubsection{Log-likelihood ratio (LLR) similarity}

LLR, one of the Mahout similarity algorithms for improving the recommendation accuracy, is a way of calculating the similarity between two objects depending on the statistics. It estimates whether the probability of overlap between preferences is coincidental or because it really is similar [66]. It is valid only when n-Grams size is greater than 1 . We used this technique as it solves the problem that, for example, while generating n-Grams, some combinations do not represent meaningful units, such as if the sentence is it was the best of days, then was the is generated in case of bigrams. When we generate weights using Td-Idf by combining the unigrams and bigrams from a text, we face many meaningless bigrams with large weights as they have large Idf. Mahout solves this problem using loglikelihood tests.

Equations 7 and 8 show the maximum likelihood estimation.

$\hat{p}\left(w_{a}\right)=\frac{c\left(w_{a}\right)}{N}$

$\hat{p}\left(w_{b} \mid w_{a}\right)=\frac{c\left(w_{a}, w_{b}\right)}{\sum_{w_{b}} c\left(w_{a}, w_{b}\right)} \approx \frac{c\left(w_{a}, w_{b}\right)}{c\left(w_{a}\right)}$

where $w$ symbolizes a phrase and $N$ denotes the total number of phrases while $c($.$) indicates the count of the$ phrase sequence in the training data [64].

\subsubsection{Normalization}

In text mining, normalization is used to transform the text into a single canonical form, namely a standard way to present the text, which ensures that the text is consistent 
before the mining operations are performed on it [67]. This process requires basic information about the type of text to be normalized and the steps of the process thereafter.

Mahout uses what is known in statistics as the $\rho$-norm for normalization [68]. For instance, the calculation the $\rho$ norm of a 2 -dimensional vector, $[a, b]$, is:

$\frac{a}{\left(|a|^{\rho}+|b|^{\rho}\right)^{1 / \rho}}, \frac{b}{\left(|a|^{\rho}+|b|^{\rho}\right)^{1 / \rho}}$

The expression $\left(|a|^{\rho}+|b|^{\rho}\right)^{1 / \rho}$ is known as the vector norm that any value greater than 0 can be used for $\rho$. To create 2-normalized bigram vectors, in short Euclidean norm, the -n flag must be set to 2 when using the seq $2 s$ parse command in Mahout. The 2-norm is demonstrated in Eq. 10 while Eq. 11 shows the infinite norm.

$$
\begin{aligned}
& \frac{a}{\sqrt{a^{2}+b^{2}}}, \frac{b}{\sqrt{a^{2}+b^{2}}} \\
& \frac{a}{\max (|a|,|b|)}, \frac{b}{\max (|a|,|b|)}
\end{aligned}
$$

where the norm power $(\rho)$ chosen depends on the type of operations performed on the vector as seen from the equations.

\section{Proposed method}

This section defines the overall architecture and implementation details of the proposed solution, HTwitt, for analysis and visualization of tweets. Figure 2 describes the high-level system architecture of proposed architecture which consists of six main components: Batch Data Injection, Data Preparation, Model Training and Testing, Real-Time Data Collection/Injection, Classification, and Visualization.

The preliminary version of this work [58] focuses Twitter data classification only on batch historical data without visualization. HTwitt is noticeably more suitable for analysis of Twitter data in terms of data collection and visualization, which enables capture of real-time streaming data at varying scales and visualization of the analyzed tweets on Google Earth. Thus, this study contributes to the background information for the use of big data frameworks in Twitter data processing.

In theory, it is a new concept to automatically determine whether tweets collected using specific keywords help to collect and visualize the information via a map. This new concept requires working with machine learning as well as big data technologies to improve the accuracy of the analysis of large amounts of tweets. The purpose of using machine learning models in the paper is to learn the different relationships between pieces of tweets using pre- labelled examples for training purposes. Then, they use these learned patterns and give the classified tweets based on the pre-defined labels without any manual intervention. The proposed work consists of four main sections. The first one is preparing raw data for model training, the second is training a Tweet classifier. The third one is the real-time collection of related tweets, and the last one is visualization of the classified tweets on Google Earth.

\subsection{Preparing raw data for model training}

The data retrieved for this work are in a complex nested JSON format that includes users' text along with around 74 different attributes, such as hashtags, coordinates, entities, truncated, name, screen_name, user_name, URLs, tweet id, etc., which needs to be processed carefully to extract the necessary information. It is very important to know the structure of the tweets to perform the pre-processing on the data.

\subsubsection{Data gathering}

The Twitter dataset acquired via the $B G S$ is used to train the model. This dataset was collected using hashtags with certain keywords, landslide, mudslide, landfall, landslip, soil sliding and without considering any specifications and location restrictions. The data was collected between 29 November 2016 and 12 March 2017. The periods when the tweets were collected are: 29 November 2016 to 20 December 2016, 13 January 2017 to 28 February 2017, and 01 March 2017 to 12 March 2017, which is in total 78 days of data. A total of nearly 100,000 tweets were collected.

\subsubsection{Pre-processing}

Preprocessing is an important step making the text more digestible by removing the noise, nonsense phrases, unnecessary repetitions so that machine learning algorithms can boost their efficiency [69]. Twitter Streaming API provides the tweets in JSON (JavaScript Object Notation) format which is the default response. However, this brings some difficulties with it, such as the size and complexity of the data. Such complex and large volume data need big data technologies in order to be processed and made ready for training and testing. In order to achieve this goal, Data Preparation component, which consists of Pre-processing and Grouping Tweets, is created. In the Pre-processing step, the necessary elements, such as country code, coordinates, the creation date, user id and text, are parsed carefully using Gson library ${ }^{4}$ used to deserialize JSON.

\footnotetext{
${ }^{4}$ https://github.com/google/gson.
} 
Fig. 2 HTwitt framework

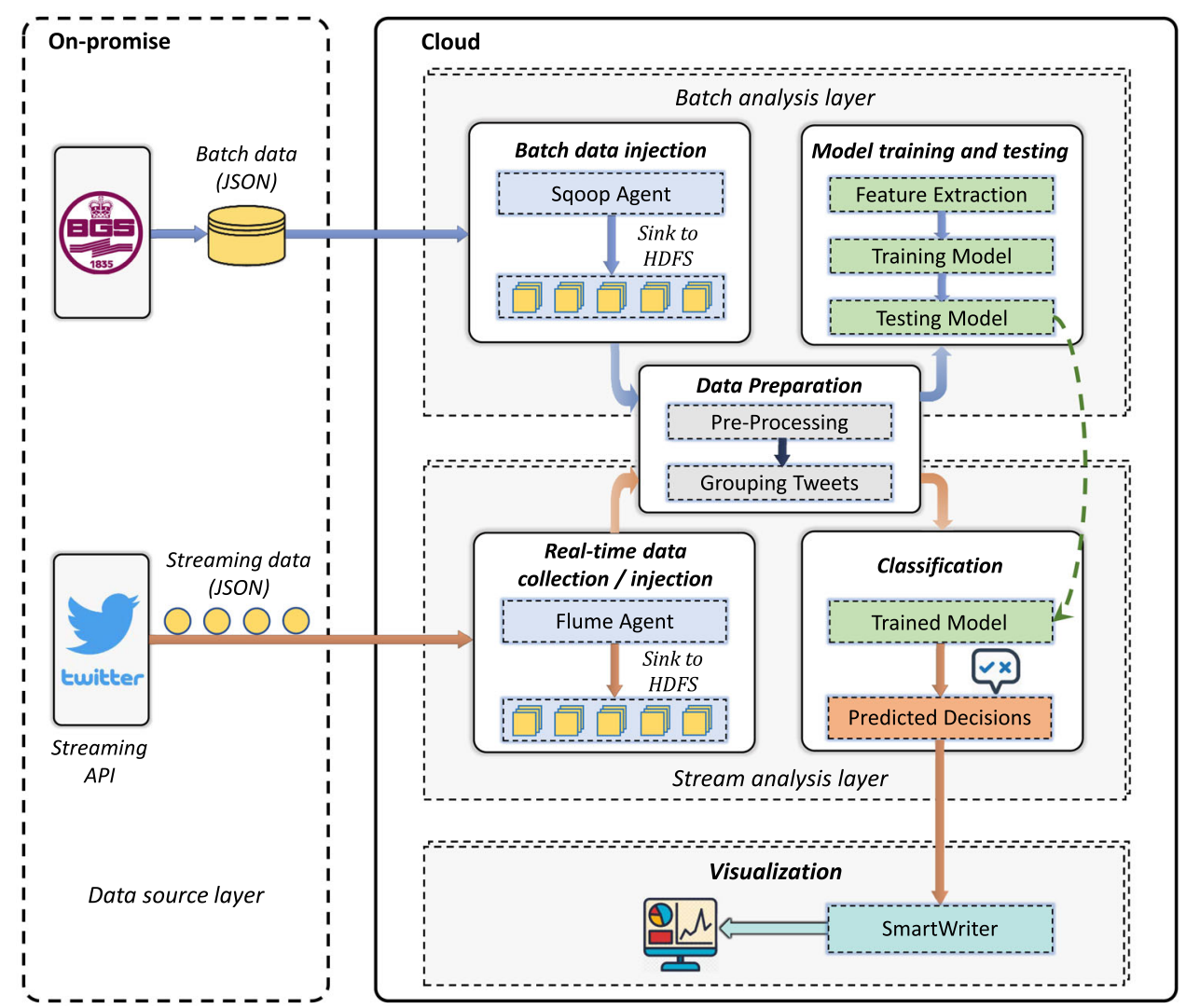

\subsubsection{MapReduce algorithm for grouping tweets}

After the parsing process, tweets are grouped depending on the unique code of each country to see which country the tweet came from and are also grouped based on the time of their creation to identify the time of events. In this way, the tweets are easily distinguished by the BGS or interested people based on these identifiers. These steps are executed using MapReduce programming model in a Hadoop cluster. Algorithm 1 and Algorithm 2 give pseudo code for Map and Reduce functions, respectively. The dataset is imported and converted into another dataset, where each element is divided into tuples (key-values) during the Map phase. As a first step, the first line of the data is taken and converted to a string value (see Algorithm 1, Line 6). Then, this value is split based on the delimiter (comma in our case) (see Algorithm 1, Line 8). Next, the keys (country code and tweet post date) are taken and are updated (see Algorithm 1, Line 10 and see Algorithm 1, Line 11). Finally, the intermediate data which is the output of the Map function are generated for the Reduce function (see Algorithm 1, Line 13).

After that, the output of the Map function is taken as input by the Reduce task and all the inputs are combined into a set of files. To this end, the key generated by the Map function is taken and a file is created and this file is entitled with this key (see Algorithm 2, Line 5). As a second step, the value generated by Map function is copied into that file (see Algorithm 2, Line 7). Besides, to group the tweets based on the date when the tweets are posted they are gathered in the same way by following Algorithm 2, Line 11 and Algorithm 2, Line 13. Lastly, the output of the Reduce function is generated and the algorithm is completed (see Algorithm 2, Line 15). The outputs serve as input for our next steps.

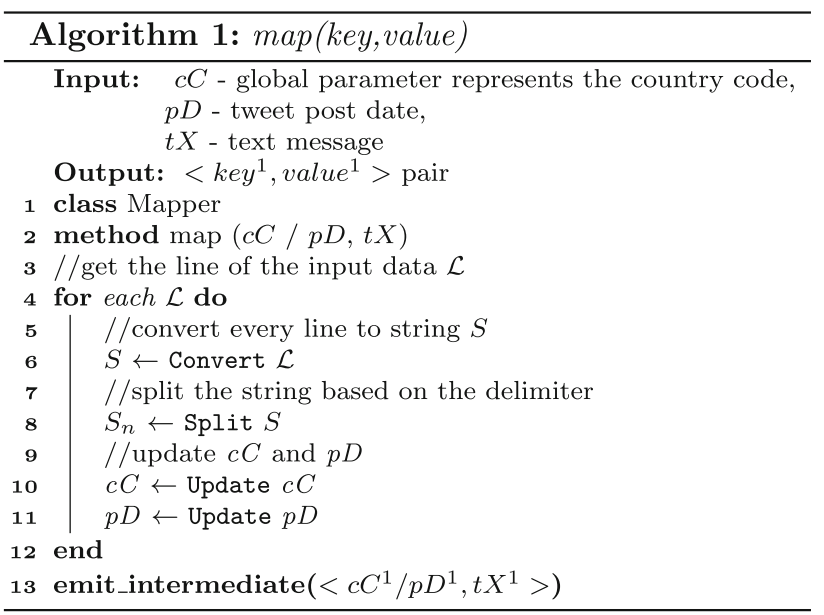




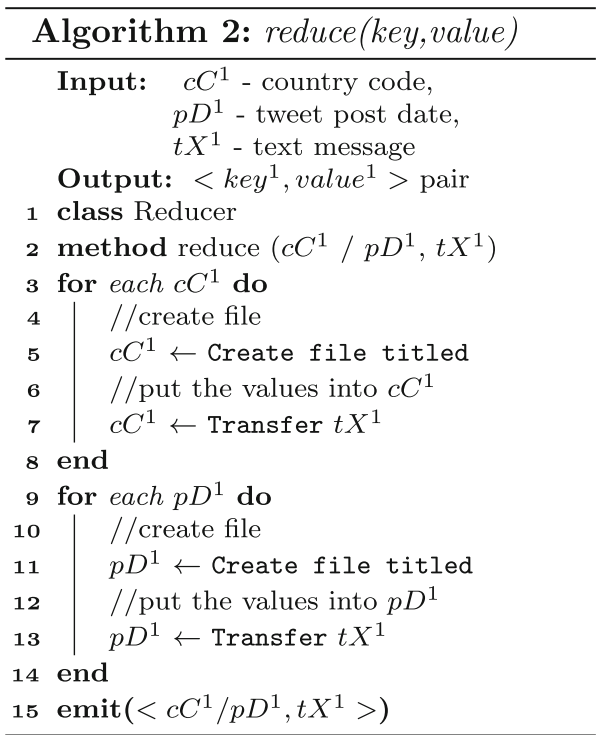

\subsection{Steps of modelling a classifier mechanism with Apache Mahout}

Apache Mahout, used for creating machine learning algorithms, implements Naïve Bayes algorithm along with three essential machine learning techniques: clustering, recommendation, and classification. We use the preliminaries mentioned in Sect. 2.1 in the Model training and testing component to model a tweet classifier. The Feature Extraction module is the key in this phase that implements the Naïve Bayes classifier. Moreover, it uses the n-Grams language model, Tf-Idf, Log-likelihood ratio similarity, and normalization technique to increase the accuracy of the classifier.

Figure 3 illustrates the phases of training the model for tweet classification in Mahout. The historical tweets are used for training the algorithm to create a model to be used for selecting the new incoming tweets in Training Model phase. After that, the model is used to automatically recognize and distinguish the tweets to use for future works, such as landslide early warning systems.

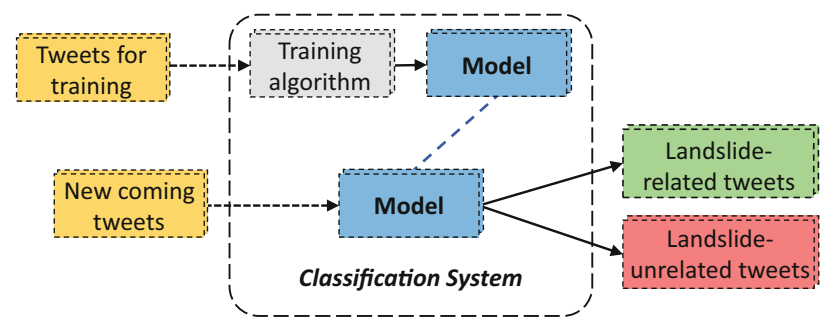

Fig. 3 Mahout working principle in HTwitt framework

\subsubsection{Feature extraction}

The Naïve Bayes classifier and n-Grams language model with Tf-Idf method are used to train a tweet classifier in this study. Before starting to create the classifier, a normalization technique is used to prepare the data for application of the machine learning. Specifically, the purpose of normalization is to transform the text into a standard form to guarantee the consistency and coherence of the text before text mining process. Naïve Bayes classifier is used in the proposed scheme as it does not require as much training data to create a model while providing a high accuracy rate. Besides, it is highly scalable and easy to use in real-time predictions. Other classifiers, such as $\mathrm{K}$-nearest neighbors $(\mathrm{KNN})$, are slower when applied to large-scale datasets as they are not linear classifiers. Similarly, decision tree performs poorly while performing big data processing. The role of the Naïve Bayes classifier in the proposed scheme is to construct a classifier model that predicts whether tweets are related to landslides using a set of calculations based on the probability principles by using the data obtained from the BGS. The n-Gram model helps to understand the occurrence of a word using the occurrence of its $\mathrm{N}-1$ previous words. We evaluate the performance of five different $n$-Gram models ranging from 1 to 5 to create the best classifier model. Tf-Idf is a numerical statistic calculated by statistical methodology showing the significance of a term in a collection or corpus, which helps the users to deduce what the author is interested in and which topics an author focuses on by examining the writings of the author. Both n-Gram model and Tf-Idf have been used to improve the accuracy in the process of creating the model.

Figure 4 shows the classifier training workflow in Naïve Bayes algorithm in Mahout. The machine learning techniques, n-Gram and Tf-Idf, are implemented in the seq2sparse phase while sequence files are being converted into vectors.

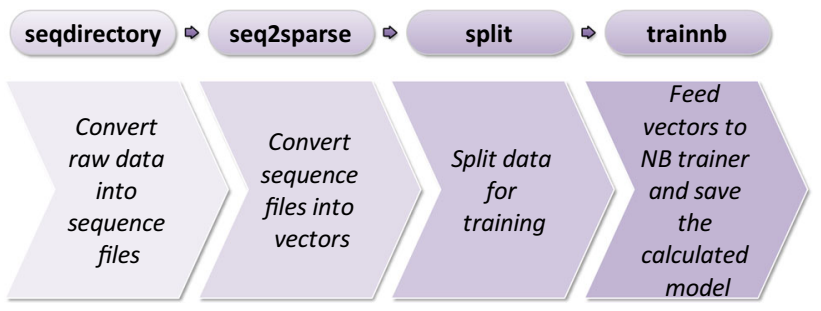

Fig. 4 Feature extraction phases in Mahout 


\subsection{Real-time collection/injection of related tweets}

In this work, the real-time data are captured from Twitter using Apache Flume via Twitter Streaming API. However, this API gives low access to entire data to developers. The data are provided in JSON format, presented in key/value pairs. The following sub-steps need to be taken for getting permission to access the streaming API to collect the streaming data and sink them into HDFS.

\subsubsection{The creating of Twitter application for Apache flume streaming}

The steps for creating a Twitter application are given below.

1. Click on https://apps.twitter.com, then sign in the Twitter account and click on "Create an app", then fill the form.

2. Create own access token, then choose what Access Type You Need.

3. Take a note of these OAuth Settings as they will be used in the Flume.conf file to connect to this application.

\subsubsection{Configuring Apache flume}

Apache Flume agent consists of three main components: Source, Sink and Channel as shown in Fig. 5. The Source pushes the events (tweets) coming through Twitter Streaming API into the Channels which buffer and store the events while Sinks retrieve the events from the Channels and sink them into HDFS. The agent needs to be configured via the configuration file Flume.conf as mentioned above. Basically, the configuration file, consisting of three main parts, enables configuration of the properties related to the source of the data, where the data will be stored, and the channel. Twitter is the source while the HDFS is used as the storage. The four tokens received from Twitter are implemented in the Source configuration part and HDFS properties are set in the Sink configuration. In

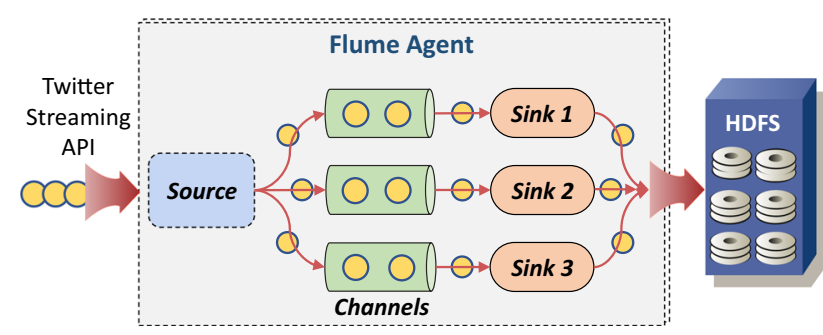

Fig. 5 The high-level architecture of Apache Flume the Source properties part, the specific keywords are stated to gather the tweets which serve the purpose of this study.

In our case, the key point of the landslide-related tweets collection is to define the keywords in the configuration file. In Flume Source section, the keywords are specified for TwitterAgent.sources.Twitter.keywords property to distinguish between the related and unrelated tweets while the collection operation. This allows to pick the tweets that include only the specific keywords before storing the events into the Channels. We set the capacity for the Channels as 10,000 which indicates the maximum number of events the Channels will take from the source using the main memory. For the Sinks, we define a location where the events coming from the Channels will be stored in HDFS and set the batch size as 1000 and set the data file format as DataStream.

\subsection{HTwitt monitoring}

Monitoring systems are commonly used to collect data from any systems to analyse their operation and performance to verify the system health [70]. In this work, SmartMonit [71] is used to monitor the performance metrics of HTwitt as well as collect infrastructure information from large-scale computer cluster in real-time. SmartMonit is implemented into the proposed system by the APIs that it provides to collect the computing resource metrics, such as CPU/memory usage, network bandwidth and disk I/O speeds using Sigar APIs, ${ }^{5}$ along with the execution status of each job and task using YARN ResourceManager REST API's. ${ }^{6}$ HTwitt runs on top of SmartMonit framework, which allows the necessary information to be collected and analyzed, while it is in progress. SmartMonit consists of a main agent called SmartAgent and two sub-agents, namely TaskAgent and SystemAgent. SmartAgent receives data from these two agents and stores it in a time-series database. TaskAgent collects the status of each task along with the cluster information processed data while SystemAgent collects the infrastructure information of the system to check the health status of each worker node in the cluster. Figure 6 shows the methodology of integration of SmartMonit and HTwitt. The performance analysis of the model will be discussed in Sect. 4.4 by referring to the total execution time required to complete all the tasks and resource utilization of the Hadoop cluster in detail.

\footnotetext{
5 https://github.com/hyperic/sigar.

${ }^{6}$ https://hadoop.apache.org/docs/r3.2.0/hadoop-yarn.
} 


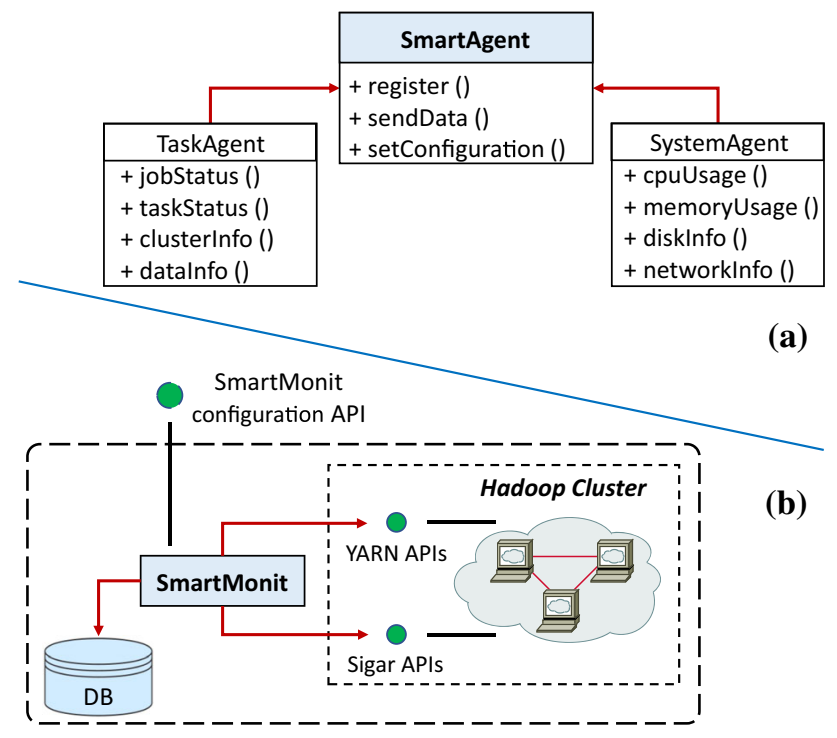

Fig. 6 Monitoring agents model (a); Implementation of SmartMonit mechanism in HTwitt (b)

\section{Experimental results and discussion}

In this section, we present the experimental results of the HTwitt to evaluate its efficiency and applicability along with its challenges in social media data analysis in cloud environments.

\subsection{Experiment setup}

We used Hadoop 3.2.0 and Apache Mahout 0.13.0 and deployed them on VM-based infrastructure over 8 AWS nodes with 1 master and 7 workers (Type: Cluster 8 in Table 2) and Ubuntu Server 18.04 LTS (HVM) was chosen as the operating system for each node. We also built eight different clusters to observe the impact of the number of worker nodes on total execution time of the model training. We only changed the number of the worker nodes while keeping the number of processors, the CPU types, speed and the memory capacity of each node unchanged to establish these clusters as shown in Table 2.

\subsection{Training and testing the model}

After making the data ready for training and testing the model, the Naïve Bayes classifier and n-Grams language model with Tf-Idf and Log-likelihood ratio similarity including normalization technique are implemented in the Model training and testing module for training the model. We used a minimum log-likelihood ratio value of 50 to include only very important bigrams. The data obtained from the BGS is used for training and testing the developed classifier module with the Naïve Bayes algorithm. First, the classifier is trained with the training data that is the sample of data used to fit the model, which is equal to $80 \%$ of the whole data set. Next, the rest of data, $20 \%$, is used for testing the classifier. The Pareto Principle, which governs the $80 / 20$ rule, is taken as a reference in determining the ratio between the sets of training and testing. The tweets grouped by country code (see Sect. 3.1.3) are used to developed the model used for classifying the newly collected tweets stored in HDFS to distinguish whether the tweet is related to a landslide event. The numbers and the rates of files used are listed in Table 3.

The classification mainly consists of two basic stages, a learning stage and the evaluation stage. In the learning stage, the model is trained with a given dataset, then the model's accuracy is evaluated depending on some performance measures.

Reliability (Rel) shows how reliably an observation is exactly the same when it is repeated, which refers to the measurement procedure instead of the measured attribute (Eq. 12), Precision $(P)$ is the degree of similarity among the results of the studies performed under similar conditions (Eq. 13), Recall $(R)$ is identified as the fraction of instances of a class estimated correctly (Eq. 14), Accuracy (A) shows how close a measurement is to the actual value (Eq. 15). Fl is defined as the weighted average of precision and recall that takes a value between 0 and 1, (Eq. 16).

$$
\begin{aligned}
& \text { Rel }=\frac{t_{s}}{t_{o}} \\
& P=\frac{T_{p}}{T_{p}+F_{p}} \\
& R=\frac{T_{p}}{T_{p}+T_{n}} \\
& A=\frac{T_{p}+T_{n}}{T_{p}+T_{n}+F_{p}+F_{n}} \\
& F_{1}=2 * \frac{T_{p}+T_{n}}{T_{p}+T_{n}+F_{p}+F_{n}}=2 * \frac{P * R}{P+R}
\end{aligned}
$$

Here, True Positive (Tp) indicates how many tweets were correctly identified as landslide-related and False Positive (Fp) states the ones detected incorrectly. True Negative (Tn) represents the number of tweets correctly detected as landslides-unrelated and False Negative (Fn) specifies the ones that state the total observed variability incorrectly while $t s$ shows the true score variability.

\subsection{Collection and sinking Twitter data using Apache flume}

After setting the configuration up, it is required to run the Flume Agent via executing the following command:

./bin/flume-ng agent / 
Table 2 Clusters specification adopted in the experiments in AWS

\begin{tabular}{lllrr}
\hline Cluster type & Specification (no. of nodes) & Total CPU Core (3.0 GHz) & Total memory (GB) & Total storage (GB) \\
\hline Cluster 1 & pseudo-distributed & 1 & 8 & 30 \\
Cluster 2 & 1 master, 1 slave & 2 & 16 & 60 \\
Cluster 3 & 1 master, 2 slaves & 3 & 24 & 90 \\
Cluster 4 & 1 master, 3 slaves & 4 & 32 & 120 \\
Cluster 5 & 1 master, 4 slaves & 5 & 40 & 150 \\
Cluster 6 & 1 master, 5 slaves & 6 & 48 & 180 \\
Cluster 7 & 1 master, 6 slaves & 7 & 56 & 210 \\
Cluster 8 & 1 master, 7 slaves & 8 & 64 & 240 \\
\hline
\end{tabular}

Table 3 The ratio of training and testing data

\begin{tabular}{lll}
\hline Data set & \\
\hline Training data $(80 \%)$ & Testing data $(20 \%)$ & Total $(100 \%)$ \\
\hline $80 \mathrm{~K}$ tweets (in 17 files) & 20K tweets (in 100 files) & 100K tweets (in 117 files) \\
\hline
\end{tabular}

-f TwitterStream.properties /

- -name TwitterAgent /

- - conf \$FLUME_HOME/conf /

-Dflume.root.logger=INFO, console

The description of the parameters of the command is given below:

name: specifies the name of the Agent. Here it is TwitterAgent.

conf: indicates the configuration directory of the flume.

Dflume.root.logger $=I N F O$, console: writes the logs to the console.

\subsection{Experimental results}

The trained model is tested for different n-Grams models at different splits of the dataset as shown in Fig. 7. The estimated accuracy results for the model indicate that the classifier with different n-Gram models accurately classifies Twitter data with an accuracy rate of around $71 \%$ to $95 \%$. The best result, 94.28\%, was reached using the 3-Grams model with $25 \%$ dataset split ratio shown in Table 4.

F1 score, the success criterion, helps to understand the performance of the classifier along with Accuracy criteria, which gives an indication of the classifier consistency. Figure 8 shows the correlation between F1 score and Accuracy based on the best results of all n-Grams values. Figure 9a demonstrates the accuracy values at five different splits of the dataset while the results of the reliability values are shown in Fig. 9b.
We have monitored the performance of our machine learning model by examining the execution time for both the training and testing phases with respect to the splits of the dataset shown in Fig. 10a and b, respectively.

The levels of overfitting in the training stage of the model via real-world data sets taken from Twitter are shown in Fig. 11a while Fig. 11b demonstrates the relationship between CPU number and execution time in the training stage of the model while the number of tweets as $80 \mathrm{~K}$ and keeping the number of Mappers unchanged. We conducted this experiment that aims to build a classifier using the 3-Grams model with $25 \%$ dataset split ratio as it has given the best results and deployed it to the eight different clusters in AWS with 3.0 GHz Intel Scalable Processors shown in Table 2.

Finally, Fig. 12a demonstrates the average CPU and memory utilization while Fig. $12 \mathrm{~b}$ shows the disk utilization across the whole cluster during the model training.

\subsection{Visualization of the classified Tweets on Google Earth}

The visualization of geographically linked data on Google Earth is one of the effective and simple ways as the user can come closer to a point of interest or go away from somewhere using a mouse. The analyzed data, containing the specific attributes, are visualized on Google Earth using the location information via SmartWriter which is the agent responsible to receive the predicted decisions and transfer them to Google Earth. Figure 13 shows the screenshots of the visualized tweets, posted from the United Kingdom that is a landslide-related tweet and posted from the United 
(a) 1-Gram model

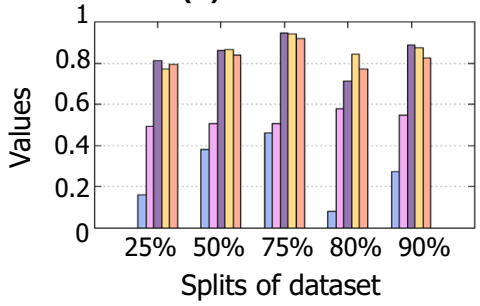

(d) 4-Grams model

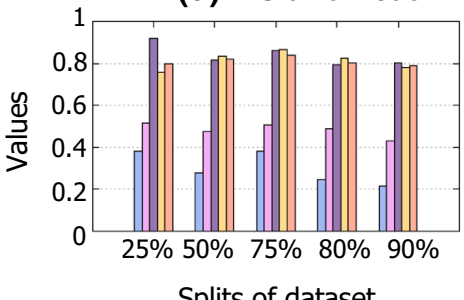

Splits of dataset (b) 2-Grams model

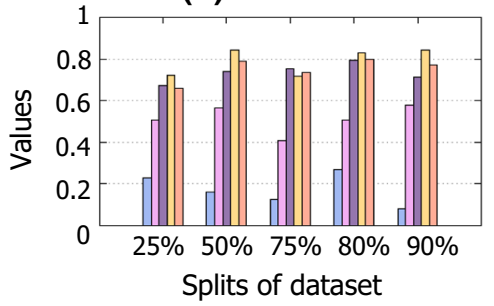

(e) 5-Grams model

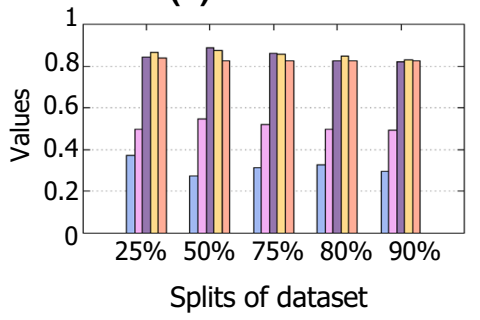

(c) 3-Grams model

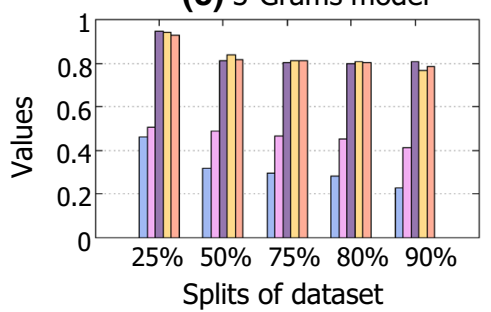

Splits of dataset

Fig. 7 The results of all n-Grams models

Table 4 The number of the classified files

\begin{tabular}{lrl}
\hline Summary & & \\
\hline Correctly classified files & 33 & $94.2857 \%$ \\
Incorrectly classified files & 2 & $5.7143 \%$ \\
Total classified files & 35 & \\
\hline
\end{tabular}

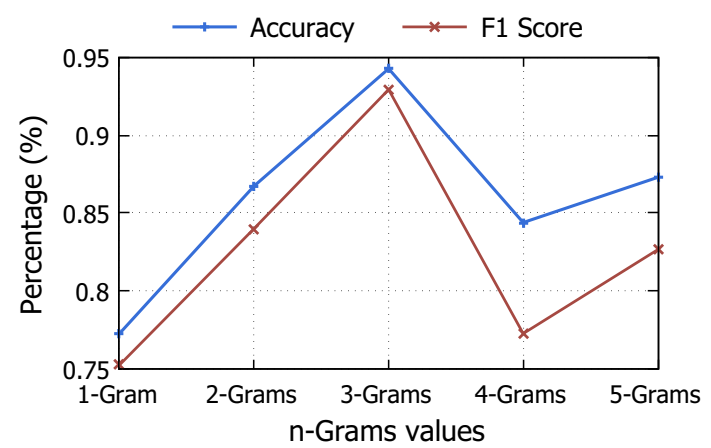

Fig. 8 Relationship between accuracy and F1 score

States that is not a landslide-related tweet, on Google Earth.

\subsection{Discussion}

Twitter data analysis has some challenges regarding data collection and processing due to the complexity of the data in JSON format. Furthermore, it requires use of efficient machine learning techniques along with big data tools to extract the beneficial and usable information from the large amount of data. Another challenge is the data collection from Twitter via Streaming API because of some restrictions set by Twitter. The main challenge is to distinguish whether the tweets are related to a landslide natural event or not due to the homonyms words. Considering these challenges, the evaluation shows the classification with Apache Mahout along with the Naïve Bayes algorithm is very efficient and the proposed framework, HTwitt, is promising, having an accuracy of $94.28 \%$.

\section{Conclusion and future work}

Many institutions and organizations around the world use social media platforms like Twitter to explore people's feelings, attitudes, and behaviors regarding any situation for mostly commercial purposes. For instance, the BGS uses Twitter to make an inference about the natural disaster of landslides. In this study, a novel technique is presented to automatically analyze tweets and a model is proposed for the BGS or those who are curious about this area to distinguish the landslide-related tweets. Apache Hadoop and Apache Mahout are used to process the given data and develop the model. The batch data from the BGS, around $100 \mathrm{~K}$, is primarily used in the training and testing stages. The results show the model is very successful in the categorization of Twitter data using big data technologies like Apache Hadoop and machine learning algorithm along with some techniques.

Combining the perspectives of batch and real-time processing techniques is a promising approach for future work. As a further step, Apache Flink could be used to collect and analyze the Twitter data in real-time as it promises high compatibility with Hadoop ecosystem as 


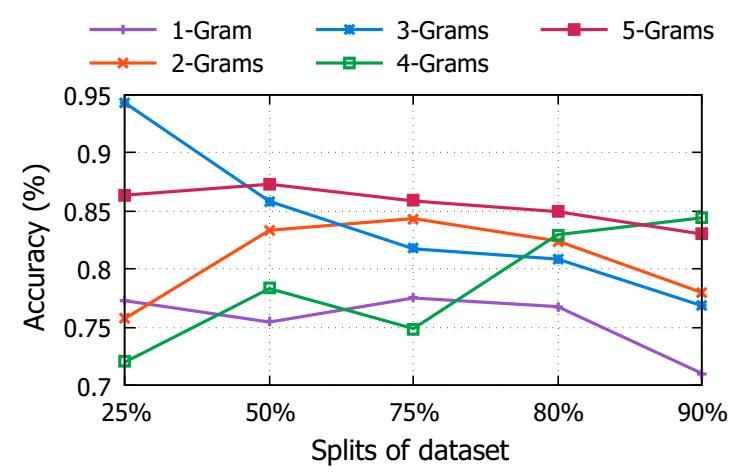

(a) Accuracy values

Fig. 9 Accuracy and reliability values at different splits values

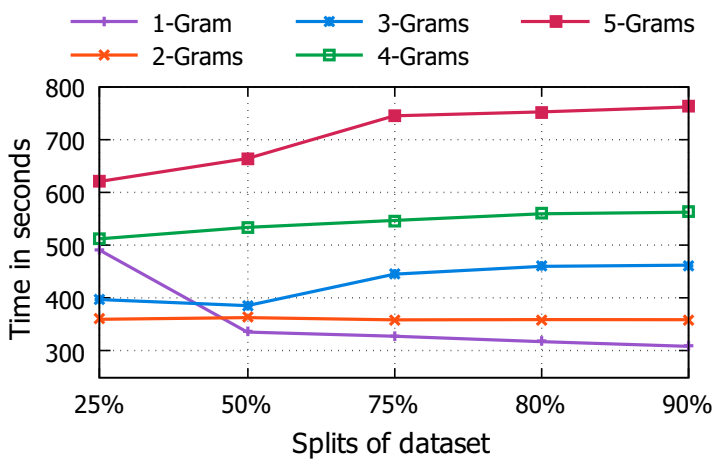

(a) Time taken for Training

Fig. 10 Time taken for all $\mathrm{n}$-Grams values

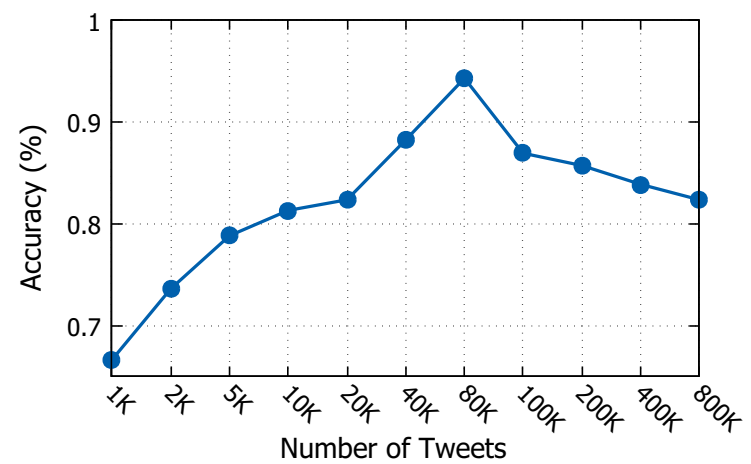

(a) The levels of overfitting in the training stage

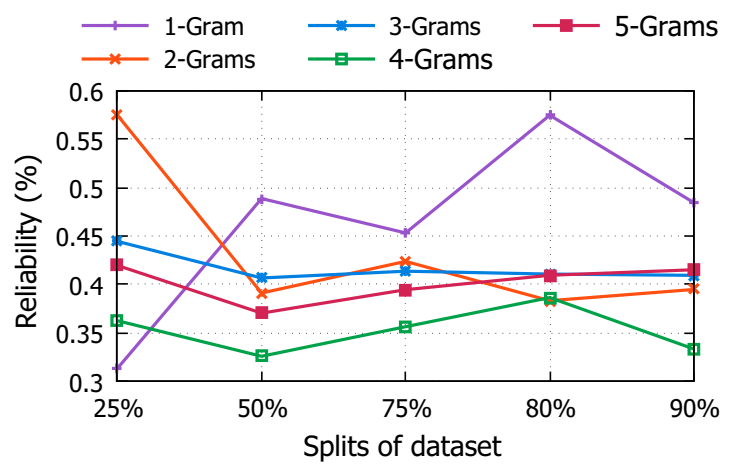

(b) Reliability valuest

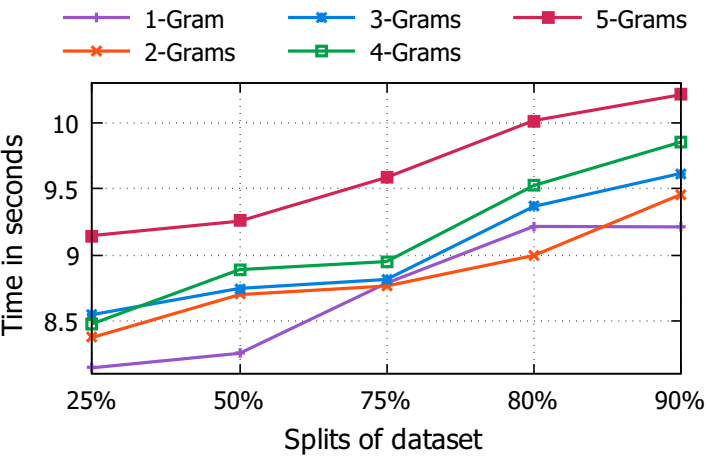

(b) Time taken for Testing

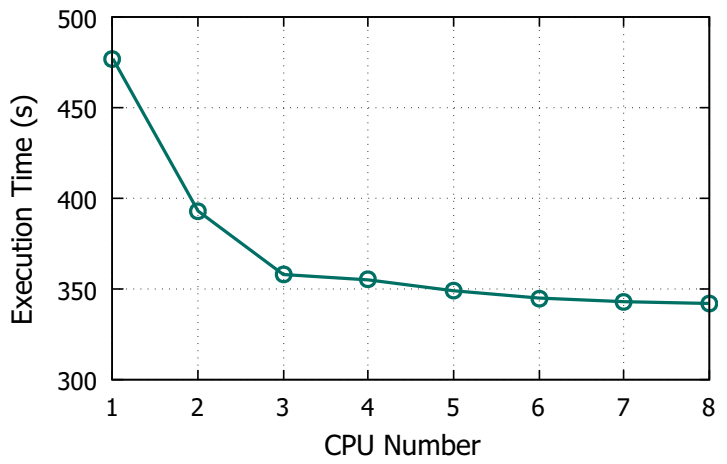

(b) Varying execution time of the model training

Fig. 11 Demonstration of the overfitting and the execution time with their dependencies

well as low latency. Deep learning techniques could be used to increase the validity of the model and natural language processing techniques with different classification methods could be used for the extraction of semantic information from Twitter data. In addition to them, using different artificial intelligence (AI) techniques, such as artificial neural networks, would increase the impact of the work. AI and machine learning (ML) techniques are gaining prominence on taking crucial decisions in early warning systems and healthcare by harnessing the power of big data. We must understand that $\mathrm{AI}$ and ML techniques 


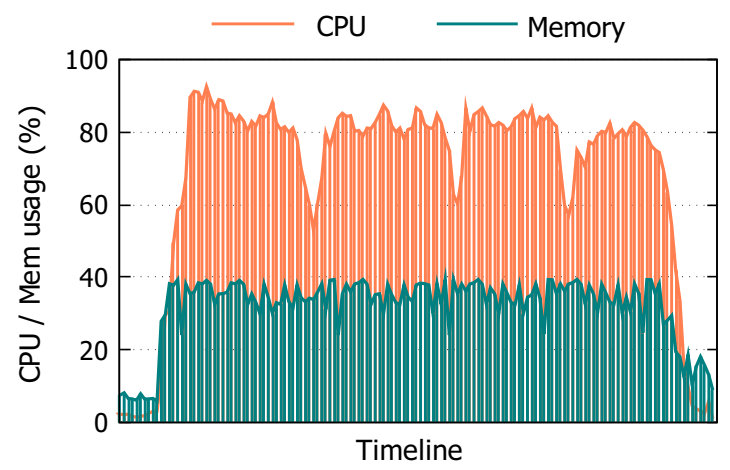

(a) Average CPU/Memory utilization

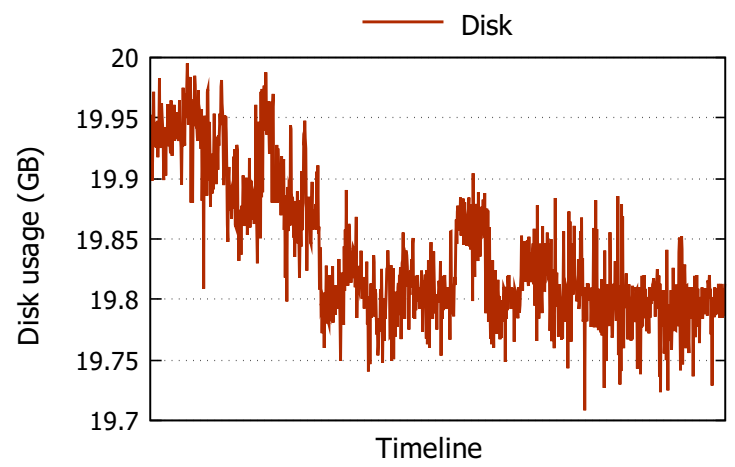

(b) Disk utilization

Fig. 12 Resource utilization of the cluster during the model training

Fig. 13 The visualized tweets on Google Earth
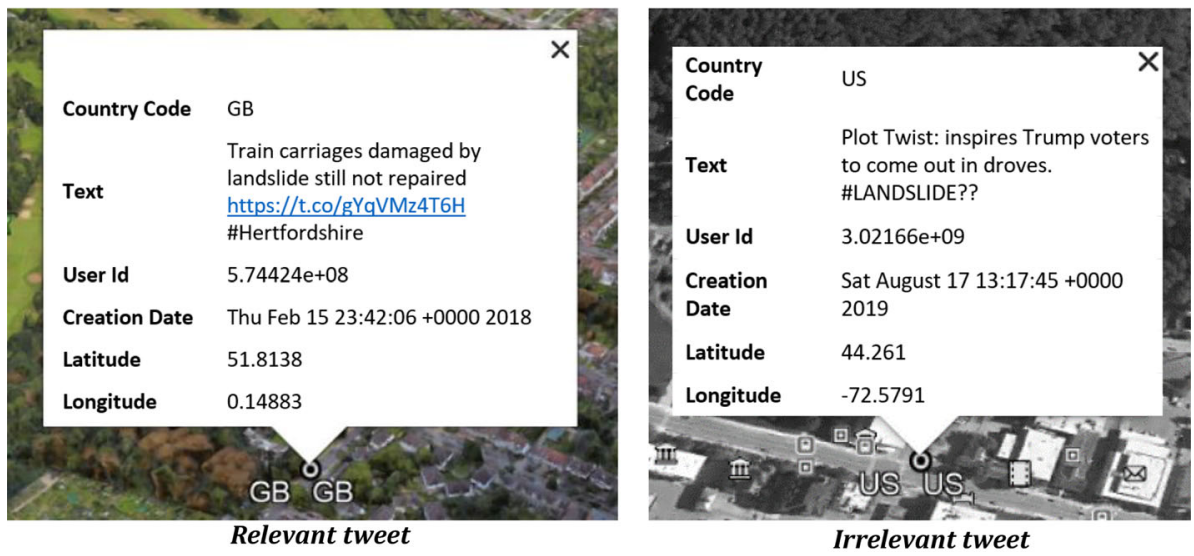

can contribute to life-saving inventions as long as applying them correctly and carefully.

Acknowledgements This research is funded by the Turkish Ministry of National Education. This research is also partially funded by the following UKRI projects including SUPER (EP/T021985/1), PACE (EP/R033293/1), and Centre for Digital Citizens (EP/T022582/1).

\section{Declarations}

Conflict of interest The authors declare that they have no conflict of interest.

Open Access This article is licensed under a Creative Commons Attribution 4.0 International License, which permits use, sharing, adaptation, distribution and reproduction in any medium or format, as long as you give appropriate credit to the original author(s) and the source, provide a link to the Creative Commons licence, and indicate if changes were made. The images or other third party material in this article are included in the article's Creative Commons licence, unless indicated otherwise in a credit line to the material. If material is not included in the article's Creative Commons licence and your intended use is not permitted by statutory regulation or exceeds the permitted use, you will need to obtain permission directly from the copyright holder. To view a copy of this licence, visit http://creativecommons. org/licenses/by/4.0/.

\section{References}

1. Luo M, Wang K, Cai Z, Liu A, Li Y, Cheang CF (2019) Using imbalanced triangle synthetic data for machine learning anomaly detection. Comput Mater Continua 58(1):15-26

2. Bukhari SA, Bashir AK, Malik KM (2018) Semantic web in the age of big data: a perspective

3. Sun L, Ge C, Huang X, Wu Y, Gao Y (2019) Differentially private real-time streaming data publication based on sliding window under exponential decay. Comput Mater Continua 58(1):61-78

4. Jindal A, Dua A, Kumar N, Das AK, Vasilakos AV, Rodrigues JJ (2018) Providing healthcare-as-a-service using fuzzy rule based big data analytics in cloud computing. IEEE $\mathrm{J}$ Biomedical and Health Inform 22(5):1605-18

5. Williams L (2019) Data DNA and diamonds. Eng Technol 14(3):62-5

6. Kim GH, Trimi S, Chung JH (2014) Big-data applications in the government sector. Commun ACM 57(3):78-85

7. Cao M, Zhou S, Gao H (2019) A recommendation approach based on product attribute reviews: improved collaborative filtering considering the sentiment polarity. Intell Autom Soft Comput 25(3):595-604

8. Zhou H, Sun G, Fu S, Jiang W, Xue J (2019) A scalable approach for fraud detection in online E-commerce transactions with big data analytics. CMC Comput Mater Continua 60(1):179-92

9. Cao N, Li S, Shen K, Bin S, Sun G, Zhu D, Han X, Cao G, Campbell A (2019) Semantics analytics of origin-destination 
flows from crowd sensed big data. CMC Comput Mater Continua 61(1):227-41

10. Sharma S, Dudeja RK, Aujla GS, Bali RS, Kumar N (2020) DeTrAs: deep learning-based healthcare framework for IoTbased assistance of Alzheimer patients. Neural Comput Appl, pp $1-13$

11. Aujla GS, Jindal A (2020) A decoupled blockchain approach for edge-envisioned IoT-based healthcare monitoring. IEEE J Sel Areas Commun 39:491-499

12. Dua A, Chaudhary R, Aujla GS, Jindal A, Kumar N, Rodrigues JJ (2018) LEASE: lattice and ECC-based authentication and integrity verification scheme in E-healthcare. In: 2018 IEEE global communications conference (GLOBECOM) 2018 Dec 9. IEEE, pp 1-6

13. Smith MD, Telang R (2016) Streaming, sharing, stealing: big data and the future of entertainment. MIT Press, Cambridge

14. Zhou K, Fu C, Yang S (2016) Big data driven smart energy management: from big data to big insights. Renew Sustain Energy Rev 56:215-25

15. Noor A, Mitra K, Solaiman E, Souza A, Jha DN, Demirbaga U, Jayaraman PP, Cacho N, Ranjan R (2019) Cyber-physical application monitoring across multiple clouds. Comput Electr Eng 77:314-24

16. Qi E, Deng M (2019) R\&D investment enhance the financial performance of company driven by big data computing and analysis. Comput Syst Sci Eng 34(4):237-48

17. Hassani H, Huang X, Silva E (2018) Digitalisation and big data mining in banking. Big Data Cogn Comput 2(3):18

18. Noor A, Jha DN, Mitra K, Jayaraman PP, Souza A, Ranjan R, Dustdar S (2019) A framework for monitoring microserviceoriented cloud applications in heterogeneous virtualization environments. In: 2019 IEEE 12th international conference on cloud computing (CLOUD) 2019 Jul 8. IEEE, pp 156-163

19. Saleem A, Khan A, Malik SU, Pervaiz H, Malik H, Alam M, Jindal A (2019) FESDA: fog-enabled secure data aggregation in smart grid IoT network. IEEE Internet Things J 7:6132-6142

20. Alwasel K, Jha DN, Habeeb F, Demirbaga U, Rana O, Baker T, Dustdar S, Villari M, James P, Solaiman E, Ranjan R (2020) IoTSim-Osmosis: a framework for modelling and simulating IoT applications over an edge-cloud continuum. J Syst Arch 28:101956

21. Phengsuwan J, Shah T, James P, Thakker D, Barr S, Ranjan R (2019) Ontology-based discovery of time-series data sources for landslide early warning system. Computing 2019:1-9

22. Jindal A, Aujla GS, Kumar N, Chaudhary R, Obaidat MS, You I (2018) SeDaTiVe: SDN-enabled deep learning architecture for network traffic control in vehicular cyber-physical systems. IEEE Netw 32(6):66-73

23. Jindal A, Aujla GS, Kumar N, Misra S (2018) Sustainable smart energy cyber-physical system: Can electric vehicles suffice its needs?. In: 2018 IEEE international conference on communications workshops (ICC workshops) 2018 May 20. IEEE, pp 1-6

24. Jindal A, Kumar N, Singh M (2020) A unified framework for big data acquisition, storage, and analytics for demand response management in smart cities. Future Gener Comput Syst 108:921-34

25. Aggarwal S, Chaudhary R, Aujla GS, Jindal A, Dua A, Kumar N (2018) Energychain: enabling energy trading for smart homes using blockchains in smart grid ecosystem. In: Proceedings of the 1st ACM MobiHoc workshop on networking and cybersecurity for smart cities 2018 Jun 25, pp 1-6

26. Jindal A, Kumar N, Singh M (2020) Internet of energy-based demand response management scheme for smart homes and PHEVs using SVM. Future Gener Comput Syst 108:1058-68

27. Gulati A, Aujla GS, Chaudhary R, Kumar N, Obaidat M, Benslimane A (2019) Dilse: lattice-based secure and dependable data dissemination scheme for social internet of vehicles. In: IEEE transactions on dependable and secure computing

28. Gu K, Wang L, Yin B (2019) Social community detection and message propagation scheme based on personal willingness in social network. Soft Comput 23(15):6267-6285

29. Grover S, Aujla GS (2014) Prediction model for influenza epidemic based on Twitter data. Int $\mathbf{J}$ Adv Res Comput Commun Eng 3(7):7541-5

30. Aujla GS, Singh A, Singh M, Sharma S, Kumar N, Choo KKR (2020) BloCkEd: blockchain-based secure data processing framework in edge envisioned V2X environment. IEEE Trans Veh Technol 69:5850-5863

31. Chaudhary R, Aujla GS, Kumar N, Rodrigues JJ (2018) Optimized big data management across multi-cloud data centers: software-defined-network-based analysis. IEEE Commun Mag 56(2):118-126

32. Kumar N, Aujla GS, Garg S, Kaur K, Ranjan R, Garg SK (2018) Renewable energy-based multi-indexed job classification and container management scheme for sustainability of cloud data centers. IEEE Trans Ind Inform 15(5):2947-57

33. Qureshi NM, Siddiqui IF, Unar MA, Uqaili MA, Nam CS, Shin DR, Kim J, Bashir AK, Abbas A (2019) An aggregate mapreduce data block placement strategy for wireless IoT edge nodes in smart grid. Wirel Pers Commun 106(4):2225-36

34. Lyubimov D, Palumbo AA (2016) Apache mahout: beyond MapReduce. CreateSpace Independent Publishing Platform

35. Zhuang X, Zhou S (2019) The prediction of self-healing capacity of bacteria-based concrete using machine learning approaches. Comput Mater Continua 59:1

36. Al-Yahya M (2019) A comparative study of machine learning methods for genre identification of classical arabic text. CMC Comput Mater Continua 60(2):421-33

37. Beck PD, Blaser M, Michalke A, Lommatzsch A (2017) A system for online news recommendations in real-time with apache mahout. InCLEF (Working Notes)

38. Tare M, Gohokar I, Sable J, Paratwar D, Wajgi R (2014) Multiclass tweet categorization using map reduce paradigm. Int $\mathrm{J}$ Comput Trends Technol (IJCTT) 9(2):78-81

39. Martín A, Julián AB, Cos-Gayón F (2019) Analysis of Twitter messages using big data tools to evaluate and locate the activity in the city of Valencia (Spain). Cities 86:37-50

40. Verma JP, Patel B, Patel A (2015) Big data analysis: recommendation system with Hadoop framework. In: 2015 IEEE international conference on computational intelligence and communication technology 2015 Feb 13. IEEE, pp 92-97

41. Yang M, Kiang M, Shang W (2015) Filtering big data from social media-building an early warning system for adverse drug reactions. J Biomed Inform 54:230-40

42. Rout JK, Choo KK, Dash AK, Bakshi S, Jena SK, Williams KL (2018) A model for sentiment and emotion analysis of unstructured social media text. Electron Commerce Res 18(1):181-99

43. Ennaji FZ, El Fazziki A, Sadgal M, Benslimane D (2015) Social intelligence framework: extracting and analyzing opinions for social CRM. In: 2015 IEEE/ACS 12th international conference of computer systems and applications (AICCSA) 2015 Nov 17. IEEE, pp 1-7

44. Khade AA (2016) Performing customer behavior analysis using big data analytics. Procedia Comput Sci 79:986-92

45. Parveen H, Pandey S (2016) Sentiment analysis on Twitter dataset using Naive Bayes algorithm. In: 2016 2nd international conference on applied and theoretical computing and communication technology (iCATccT) 2016 Jul 21. IEEE, pp 416-419

46. Nodarakis N, Sioutas S, Tsakalidis AK, Tzimas G (2016) MRSAT: a MapReduce algorithm for big data sentiment analysis on Twitter. InWEBIST (1) 2016 Apr 23, pp. 140-147 
47. Yan P (2017) Mapreduce and semantics enabled event detection using social media. J Artif Intell Soft Comput Res 7(3):201-13

48. Chunne AP, Chandrasekhar U, Malhotra C (2015) Real time clustering of tweets using adaptive PSO technique and MapReduce. In: 2015 global conference on communication technologies (GCCT) 2015 Apr 23. IEEE, pp 452-457

49. Ed-Daoudy A, Maalmi K (2019) Real-time machine learning for early detection of heart disease using big data approach. In: 2019 international conference on wireless technologies, embedded and intelligent systems (WITS) 2019 Apr 3. IEEE, pp 1-5

50. Alotaibi S, Mehmood R, Katib I, Rana O, Albeshri A (2020) Sehaa: a big data analytics tool for healthcare symptoms and diseases detection using Twitter, apache spark, and machine learning. Appl Sci 10(4):1398

51. Kumar A, Singh M, Pais AR (2019) Fuzzy string matching algorithm for spam detection in Twitter. In: International conference on security and privacy (2019 Jan 9). Springer, Singapore, pp 289-301

52. Kafeza E, Kanavos A, Makris C, Pispirigos G, Vikatos P (2019) T-PCCE: Twitter personality based communicative communities extraction system for big data. IEEE Trans Knowl Data Eng 32:1625-1638

53. El Abdouli A, Hassouni L, Anoun H (2019) A distributed approach for mining moroccan hashtags using Twitter platform. In: Proceedings of the 2nd international conference on networking, information systems and security 2019 Mar 27, pp 1-10

54. Tripathi AK, Sharma K, Bala M, Kumar A, Menon VG, Bashir AK (2020) A parallel military dog based algorithm for clustering big data in cognitive industrial internet of things. IEEE Trans Ind Inform 17:2134-2142

55. Wang G, Liu M (2019) Dynamic trust model based on service recommendation in big data. Comput Mater Continua 58:845-857

56. Chinnov A, Kerschke P, Meske C, Stieglitz S, Trautmann H (2015) An overview of topic discovery in Twitter communication through social media analytics

57. Barika M, Garg S, Zomaya AY, Wang L, Moorsel AV, Ranjan R (2019) Orchestrating big data analysis workflows in the cloud: research challenges, survey, and future directions. ACM Comput Surv (CSUR) 52(5):1-41

58. Demirbaga U, Jha DN (2018) Social media data analysis using MapReduce programming model and training a Tweet Classifier using Apache Mahout. In: 2018 IEEE 8th international symposium on cloud and service computing (SC2) 2018 Nov 18. IEEE, pp 116-121
59. Ramaraju R, Ravi G, Madhavi K (2021) Sentimental analysis on Twitter data using hadoop with spring web MVC. In: Intelligent system design. Springer, Singapore, pp 265-273

60. Dulhare UN, Gouse S (2020) Hands on MAHOUT-machine learning tool. Mach Learn Big Data Concepts Algorithms Tools Appl 15:361-421

61. Vohra D (2016) Apache flume. In: Practical Hadoop ecosystem 2016. Apress, Berkeley, CA, pp 287-300

62. Rish I (2001) An empirical study of the Naive Bayes classifier. In: IJCAI 2001 workshop on empirical methods in artificial intelligence 2001 Aug 4, vol 3, No 22, pp 41-46

63. Brown PF, Desouza PV, Mercer RL, Pietra VJ, Lai JC (1992) Class-based n-gram models of natural language. Comput Linguist 18(4):467-79

64. Siu M, Ostendorf M (2000) Variable n-grams and extensions for conversational speech language modeling. IEEE Trans Speech Audio Process 8(1):63-75

65. Aizawa A (2003) An information-theoretic perspective of tf-idf measures. Inf Process Manag 39(1):45-65

66. Ahmad M, Bashir AK, Khan AM (2017) Metric similarity regularizer to enhance pixel similarity performance for hyperspectral unmixing. Optik 140:86-95

67. Ujjan RM, Pervez Z, Dahal K, Bashir AK, Mumtaz R, González J (2019) Towards sFlow and adaptive polling sampling for deep learning based DDoS detection in SDN. Future Gener Comput Syst 111:763-779

68. Owen S, Owen S (2012) Mahout in action. Manning, Shelter Island

69. Qian B, Su J, Wen Z, Jha DN, Li Y, Guan Y, Puthal D, James P, Yang R, Zomaya AY, Rana O (2020) Orchestrating the development lifecycle of machine learning-based IOT applications: a taxonomy and survey. ACM Comput Surv (CSUR) 53(4):1-47

70. Aujla GS, Barati M, Rana O, Dustdar S, Noor A, Llanos JT, Carr M, Marikyan D, Papagiannidis S, Ranjan R (2020) COM-PACE: compliance-aware cloud application engineering using blockchain. IEEE Internet Comput 24(5):45-53

71. Demirbaga U, Noor A, Wen Z, James P, Mitra K, Ranjan R (2019) SmartMonit: real-time big data monitoring system. In: 2019 38th symposium on reliable distributed systems (SRDS) 2019 Oct 1. IEEE, pp 357-3572

Publisher's Note Springer Nature remains neutral with regard to jurisdictional claims in published maps and institutional affiliations. 